\title{
Prevalence of risk factors and
}

\section{asymptomatic carotid atherosclerosis in diabetic patients screened for silent myocardial ischemia by SPECT myocardial imaging}

Irena Peovska Mitevska ${ }^{1}$, Natalija Baneva², Marijan Bosevski ${ }^{1}$, Elizabeta Srbinovska Kostovska ${ }^{1}$

'University Cardiology Clinic

${ }^{2}$ Neurology Clinic, Medical Faculty, University "St Cyril and Methodius" Skopje, Macedonia

[Received 8 I 2016; Accepted 20 VIII 2016]

\begin{abstract}
BACKGROUND: The aim of the study was to evaluate whether there is any association between myocardial ischemia, common risk factors and carotid artery ultrasound parameters in asymptomatic type 2 diabetic (DMT2) patients.

MATERIAL AND METHODS: 60 asymptomatic DMT2 patients (pts) without known coronary artery disease (CAD) underwent one day rest Dypiridamole stress Tc-99m sestamibi single photon emission computed tomography myocardial perfusion scintigraphy (MPS). We used 17 segment models for perfusion analysis with the assessment of perfusion scores. Patients were studied for age, sex, hypertension, hyperlipidemia, hs-CRP, smoking, obesity and family history of cardiac disease. Color Ultrasound examination of carotid arteries was performed in all patients.

RESULTS: 51 patients (pts) had hypertension, 48 pts had hyperlipidemia, 15 were smokers, 6 pts had BMl $>30 \mathrm{~kg} / \mathrm{m}^{2}$ and 26 patients had positive family history for CAD. 18 (31\%) patients had myocardial ischemia. Mild ischemia was found in 6 pts, moderate in 7 patients and severe ischemia in 5 patients. Carotid IMT was increased in 34 pts and 15 pts had carotid plaques. Mean C-IMT value in patients with normal MPS results was $0.7 \pm 0.1$; in moderate ischemia $0.9 \pm 0.1$ and in pts severe ischemia $1.0 \pm 0.2$. Multivariate analysis showed obesity, low HDL and increased diastolic blood pressure predictors of increased c-IMT. Increased pulse pressure (PP), age and non-HDL cholesterol were predictors for presence of carotid plaques. Multivariable analysis for prediction of stress induced ischemia showed OR 2.9 (95\% Cl 2.15.1) for male gender, OR 3.1 for systolic blood pressure (95\% Cl 1.9-3.8) and OR 2.8 for LDL cholesterol (95\% Cl 1.7 3.6).

CONCLUSIONS: Our study has shown high prevalence of traditional risk factors and silent myocardial ischemia in type 2 diabetic patients, with the importance of SPECT imaging in selected diabetes type 2 patients. The study highlights the importance of screening for carotid atherosclerosis, which may be useful to identify diabetic patients at higher risk for coronary artery disease..
\end{abstract}

KEY words: myocardial perfusion scintigraphy, carotid artery disease, myocardial ischemia, risk factors

Nucl Med Rev 2017; 20, 1: 3-9

\section{Background}

Type 2 diabetes (DMT2) is an established risk factor for increased cardiovascular events. A two- to four-fold increased risk of CVD in people with diabetes compared with non-diabetic pop-

Correspondence to: Irena Peovska Mitevska

University Cardiology Clinic

Ul. Vodnjanska 17, 1000 Skopje, Macedonia

Tel: + 38975491216

E-mail: peovskai@yahoo.com ulation has been reported by various studies [1, 2]. The scientific literature data estimates the prevalence of silent myocardial ischemia (SMI) in DMT2 between 15-59\%. Approximately $40 \%$ of the DMT2 population had silent myocardial infarction, which might lead to sudden cardiac death [3, 4]. There was a long debate regarding the best way to screen asymptomatic diabetic patients for significant CAD [4]. Diabetes has been defined as an independent factor for progression of atherosclerosis, measured by rise in carotid intima-media thickness (c-IMT), but also for the presence of high grade carotid stenosis. Diabetes is linked to endothelial dysfunction, insulin resistance, 
vascular inflammation, diffuse atherosclerosis and increased thrombotic risk. Although atherosclerosis is a pathological polivascular process, a non-invasive ultrasonography evaluation of carotid arteries enables more precise estimate of the overall progression of atherosclerosis and improves cardiovascular risk profile. It was previously reported that C-IMT is closely related to the incidence of cerebrovascular disease and CAD in elderly subjects and diabetic patients as well $[5,6]$. There are not many studies assessing the relationship of risk factors, increased c-IMT, carotid plaques and presence of myocardial ischemia in diabetic patients. The aim of this study was to evaluate the risk factor profile, presence of asymptomatic carotid artery disease and predictors of CAD in a group of asymptomatic DMT2 patients screened for myocardial ischemia.

\section{Material and methods}

\section{Study design and protocol}

Our study is designed as a nonrandomized cross sectional clinical study. The study evaluated 60 consecutive asymptomatic patients with DMT2 (34 male, 26 female; age range of $67 \pm 6$ years), without previously known or established CAD were included in the study. All patients filled out the World Health Organization (WHO) Rose Angina Questionnaire for confirmation of the asymptomatic status. Screening for myocardial ischemia was performed using MPI SPECT imaging in the nuclear cardiology laboratory at our clinic. Color Doppler ultrasound of carotid arteries was performed in the vascular lab by experienced physicians, who were not aware of patient's history and MPI findings.

This study is as a part of the doctoral research thesis data, approved by the Ethical Committee of Medical Faculty, University St. Cyril and Methodius in Skopje, Macedonia.

Physical examination was performed in all patients which also included blood pressure measurement, weight, height, body mass index (BMI) and risk factors analysis. Full blood laboratory analyses with HDL, LDL, non-HDL and triglyceride, fasting glucose levels, HbA1C (\%) levels were performed within a maximum of 2 weeks prior to MPI study. Clinical and laboratory characteristics are shown in Table 1. The following inclusion criteria were used: asymptomatic patients with DMT2; exclusion criteria were typical stable angina pectoris, previously known or established as CAD (history of myocardial infarction, acute coronary syndromes, previous percutaneous intervention or coronary artery bypass surgery), LVEF $<50 \%$ at rest, severe valvular disease, atrial fibrillation, left bundle branch block, presence of pace maker, severe chronic pulmonary disease.

Physical examination and full disease history was taken on the day of MPS performance. Blood pressure was measured after 5 minutes rest in sitting position in all patients. Pulse pressure (PP) was calculated as PP = systolic blood pressure (SBP) - diastolic blood pressure (DBP). Laboratory examination was performed in all patients $2 \pm 1$ week before MPS study and it included the following parameters: full blood count, fasting glucose level, $\mathrm{HbA} 1 \mathrm{c} \%, \mathrm{BUN}$, creatinin, electrolytes - sodium and potassium, AST, ALT, fasting lipids values-cholesterol, LDL, HDL, triglyceride, non-HDL cholesterol. High sensitive CRP (hs-CRP) was evaluated quantitatively with high sensitivity assay, imunoturbidimetric Abbot ARCHITECT c8000 analyzer, with analytical sensitivity $0.15-15 \mathrm{mg} / \mathrm{l}$. The following
Table 1. Clinical characteristics of the study population

\begin{tabular}{lcc} 
Variables & Mean values & $\begin{array}{c}\text { Standard } \\
\text { deviation }\end{array}$ \\
\hline Systolic pressure [mm Hg] & 154.9 & 17.3 \\
Diastolic pressure [mm Hg] & 85.3 & 8.9 \\
Pulse pressure [mm Hg] & 65 & 11.0 \\
Weight [kg] & 83.5 & 12.2 \\
Height [cm] & 171.6 & 6.2 \\
BMI [kg/m²] & 27.3 & 3.6 \\
Total cholesterol [mmol/L] & 5.5 & 1.3 \\
HDLcholesterol [mmol/L] & 0.9 & 0.5 \\
Non-HDLcholesterol [mmol/L] & 4.1 & 1.2 \\
LDLcholesterol [mmol/L] & 3.6 & 1.1 \\
Triglycerides [mmol/L] & 2.2 & 0.9 \\
hs-CRP & 2.1 & 1.5
\end{tabular}

BMI — body mass index; HDL — high-density lipoprotein; LDL — low-density lipoprotein

hs-CRP values were used for CV risk prediction: $<1 \mathrm{mg} / \mathrm{L}-$ low risk; $1-3 \mathrm{mg} / \mathrm{L}$ moderate risk; $>3 \mathrm{mg} / \mathrm{L}$ high risk.

\section{Definition of study variables}

Risk factor definitions were made according to the most recent ESC guidelines on hypertension, hyperlipidemia and cardiovascular prevention: arterial hypertension (systolic blood pressure (SBP) $>140 \mathrm{bpm}$ or diastolic BP > $90 \mathrm{bpm}$ ), hyperlipidemia (LDL > $1.8 \mathrm{mmol} / \mathrm{l}$ for diabetic patients; high-density lipoprotein $<1.1$ and $1.0 \mathrm{mmol} / \mathrm{l}$ for women and man respectively and triglycerides $>1.7 \mathrm{mmol} / \mathrm{l})$, family history ( $\mathrm{Ml}$ or sudden cardiac death in an immediate male relative $<55$ years or female $<65$ years), smoker (current smoker or those who quit in the past 6 months). BMl $\geq 30 \mathrm{~kg} / \mathrm{m}^{2}$ was used to define obese patients. Type 2 diabetes mellitus was defined as established disease in patients treated with oral antidiabetic medication or insulin following initial treatment with oral anti-diabetic therapy. Newly diagnosed diabetes was defined as having either one of the following criteria, base on the ESC guidelines on prediabetes and diabetes: fasting glucose of $7.0 \mathrm{mmol} / \mathrm{L}$ or non-fasting glucose $11.0 \mathrm{mmol} / \mathrm{L}$ in 2 separate samples, $\mathrm{HbA} 1 \mathrm{c}>6.5 \%$ or pathologic OGTT in patients with fasting glucose over 6.5 without previously known or treated diabetes.

\section{Myocardial perfusion SPECT imaging (MPI)}

MPI SPECT imaging was performed using one day rest stress protocol with radiotracer Tc-99m sestamibi, using $15 \mathrm{mCl}$ for the rest and $25 \mathrm{mCl}$ for the stress study. We used single head gamma camera Siemens e-cam, with commercially available quantitative gated and perfusion SPECT software package (4DM-SPECT). Patients were instructed to refrain from caffeine-containing beverages for at least $12 \mathrm{~h}$, nitrates for $24 \mathrm{~h}$ and beta-blockers for $48 \mathrm{~h}$ before the study. All patients underwent pharmacological stressing with Dipyridamole. We used the 17-segment model for quantitative Bull's eye analysis of regional myocardial perfusion and function. Myocardial perfusion was assessed by 5 point score system (0 - normal radiotracer uptake; 1 - mild, 2 - moderate; 3 - severe hypoperfusion; 4 - absent uptake). Semi quantitative analysis of regional perfusion at rest and stress was performed 
Table 2. Clinical characteristics of patients with and without myocardial ischemia

\begin{tabular}{|c|c|c|c|c|}
\hline Variables & $\begin{array}{c}\text { Patients } \\
n=60\end{array}$ & $\begin{array}{c}\text { MPI ischemia } \\
n=18\end{array}$ & $\begin{array}{c}\text { Normal MPI } \\
n=42\end{array}$ & $p$ value \\
\hline Age & $65 \pm 6$ & $69 \pm 3$ & $64 \pm 2$ & $<0.05$ \\
\hline Gender & $34 \mathrm{~m} / 26 \mathrm{f}$ & $41 \mathrm{~m} / 19 \mathrm{f}$ & $36 \mathrm{~m} / 24 \mathrm{f}$ & $<0.01$ \\
\hline Hypertension & 45 (76\%) & 28 & 17 & $<0.001$ \\
\hline Hyperlipidemia & 31 (51.6\%) & 20 & 11 & $<0.01$ \\
\hline Family hist CAD & 26 (43.3\%) & 12 & 13 & 0.071 \\
\hline Active smoker & 8 (13.3\%) & 4 & 4 & 0.079 \\
\hline
\end{tabular}

CAD — coronary artery disease; MPI — myocardial perfusion imaging

using summed stress score (SSS), summed rest score (SRS) and summed differential score (SDS), aimed to assess the presence and extend of myocardial ischemia. Scan abnormalities were defined as follow: SSS $<4$ normal perfusion; 4-8 mild; 9-13 moderate; $>13$ severely abnormal scan. SDS $<6$ mild $(<10 \%$ of LV); SDS $7-10$ moderate $(10-15 \%$ of LV); SDS $>10$ severe ischemia $(>15 \%$ of LV). Any perfusion abnormality was defined as SDS $>4$ and/or SRS $>4$. Regional wall motion analysis was assessed by 6 point scoring system at rest and stress $(0-$ normal wall motion, 1 - mild, 2 - moderate; 3 - sereve hypokinesia, 4 - akinesia, 5 - dyskinesia) using wall motion score index.

\section{Color Doppler carotid ultrasound}

All patients underwent a two-dimensional echo-color Doppler examination of carotid arteries bilaterally using a high-definition vascular echo machine (Philips HD11XE). The Mannheim carotid intima-media thickness consensus (2004/2006/2011) defined evaluation criteria of intima-media thickness (IMT) and atherosclerotic plaques. IMT was measured in $10 \mathrm{~mm}$ segment at end diastole, proximal from carotid bulbe in the internal carotid artery, carotid bifurcation and the proximal part of the internal carotid artery bilaterally. Carotid IMT $>0.8 \mathrm{~mm}$ was defined as abnormal. The presence of atherosclerotic plaques can be diagnosed by detection of an IMT $>1.3 \mathrm{~mm}$ or a focal structure emerging from the wall of at least $0.5 \mathrm{~mm}$ or $50 \%$ of the surrounding IMT value. Carotid stenosis greater than $60 \%$ was considered significant.

\section{Statistical analysis}

Statistical analysis was performed with the use of the SPSS statistical package (version 18.0). Categorical variables were compared using chi-square test and continuous variables using unpaired Student t test. Categorical values were expressed in percentages, continued as mean value $\pm \mathrm{SD}$. We applied the Pearson method for correlations assessment. Linear regression analysis was used to determine whether there was a correlation between the carotid ultrasound findings and MPI perfusion abnormalities. Multivariate forward stepwise logistic regression analysis was built in order to identify factors associated independently with the presence of silent myocardial ischemia and carotid atherosclerosis. The analysis includes inflammation biomarker hs-CRP, clinical risk factors and carotid ultrasound parameters. The prevalence of mild ischemia (SDS 4-7) and severe ischemia (SDS > 7) was compared between patients with normal and increased c-IMT. The criterion for entrance into the model was a univariate probability value of $p<0.05$ and $p>0.10$ for removal from the model. $P<0.05$ was found to be statistically significant for all statistical tests.

\section{Results}

Studied population consisted of 34 male (56.6\%) and 26 female patients (43.3\%), with mean age of $65 \pm 6$ years, respectively, as shown in Table 1. The prevalence of traditional risk factors and laboratory findings are presented in Table 1 and 2. All patients had on average 2 risk factors. The risk factors for the cardiovascular disease were distributed as follows: $76 \%$ of systemic arterial hypertension; $51.6 \%$ of hyperlipidemia; $13.3 \%$ of cigarette-smoking, $11.6 \%$ with obesity and $43.3 \%$ of family history for coronary history. Average duration of diabetes was $8 \pm 3$ years. 11 patients (18.3\%) used insulin therapy. The average hs-CRP values were $2.1 \pm 1.5 \mathrm{mg} / \mathrm{L}$.

\section{Myocardial SPECT findings}

The stress phase was carried out through pharmacological stress with dipyridamole in all patients. Total of 1020 segments were analyzed. All patients hadnormal rest left ventricular function with EF $>55 \%$ assessed by Gated SPECT. The scintigraphy results were abnormal in 21 patients (35\%). Stress inducible ischemia was found in 18 pts (30\%). Mild ischemia was found in 6 patients (10\%) - summed stress score (SDS) $<4$, moderate ischemia (SDS $7-10)$ in 7 patients (11.6\%) and severe ischemia (SDS > 10) in 5 patients (8.3\%) (Figure 1). Two patients had reversed perfusion defects and one patient fixed perfusion defect in one segment.

We compared scintigraphy data against common cardiovascular risk factors. We found significant correlation between degree of myocardial ischemia and mean value of c-IMT $(r=0.62$, $p<0.001)$, hyperlipidemia $(r=0.69, p<0.001)$ and systolic BP $(r=0.52, p<0.05)$. We found that the patients with abnormal MPI result were mainly older male population $(p=0.01)$, comparing to the group with normal MPI results. Among the 18 patients with scintigraphy myocardial ischemia findings, all had at least 2 risk factors: 10 (55.5\%) had 2 risk factors, 6 (33.3\%) had 3 risk factors and $2(11.1 \%)$ had 4 risk factors. Among the 42 patients with normal scintigraphy: 25 (59.5\%) had 2 risk factors, 15 (35.7\%) had 3 risk factors. Association between moderate and severe ischemia and C-IMT is shown in Figure 2. 


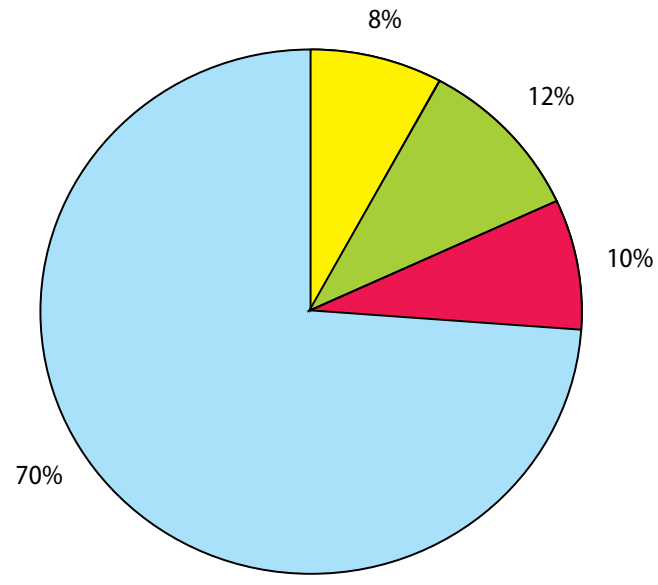

Normal

Mild ishemia

Figure 1. Scintigraphic findings in studied population

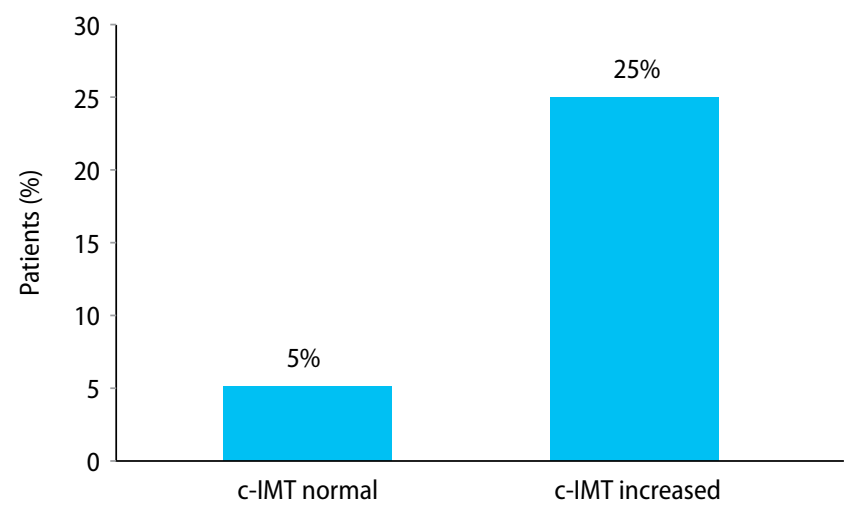

Figure 2. Association between moderate and severe ischemia and c-IMT

\section{Multivariable analysis for prediction of myocardial ischemia}

We have performed initial univariate analysis for prediction of myocardial ischemia in DMT2 patients which includes the following parameters: age, gender, systolic BP, diastolic BP, pulse pressure, cholesterol, LDL, HDL, triglycerides, non-HDL, BMI > $30 \mathrm{~kg} / \mathrm{m}^{2}$, smoking, hs-CRP, family history of CAD, mean c-IMT, presence of carotid plaques. After adjustment for age, male gender, LDL cholesterol and hypertension in multivariate model, increased $c$-IMT remained a significant predictor of SDS ( $p<0.001$ ) (OR 3.811 [95\% Cl 2.251-5.963]). Multivariate analysis showed male gender, systolic blood pressure, LDL cholesterol and increased mean c-IMT independent predictors for presence of myocardial ischemia (Table 3)

\section{Carotid ultrasound findings}

Clinical characteristics of the study cohort in relation to the carotid ultrasound findings are shown in Table 4. Normal C-IMT $(<0.8 \mathrm{~mm})$ was found in 26 patients $(43.3 \%)$, while in the remaining 34 patients (56.5\%), c-IMT was present in one of the common carotid arteries. The majority of patients with increased c-IMT and carotid plaques were older pts with hypertension and hyperlipidemia.

\section{$c-I M T$ findings in relation to the extent of SPECT myocardial ischemia}

We found the increase in mean c-IMT parallel with the degree of myocardial ischemia (Table 5). The mean SDS increased from $2.4 \pm 1.1$ in patients with normal c-IMT to $8.5 \pm 1.3$ in patients with increased c-IMT $(p<0.001)$. Results of multivariate regression analysis are shown above, with c-IMT remained a significant predictor of myocardial ischemia in the multivariate model $(p<0.001)$.

\section{c-IMT findings in relation to prevalence of abnormal SPECT results}

We found abnormal myocardial perfusion results in $11 \%$ of patients with normal c-IMT. In contrast, $81 \%$ of patients with abnormal MPI results and myocardial ischemia findings had increased c-IMT.

Table 4. Clinical characteristics of the study population in relation to carotid ultrasound findings

\begin{tabular}{lccc} 
Variables & IMT $<0.8$ & IMT $>0.8$ & Plaques \\
& $\mathbf{n}=26$ & $\mathrm{n}=34$ & $\mathrm{n}=15$ \\
\hline Age & $69 \pm 4$ & $64 \pm 4$ & $68 \pm 2$ \\
Gender & $24 \mathrm{~m} ; 16 \mathrm{f}$ & $24 \mathrm{~m} ; 16 \mathrm{f}$ & $11 \mathrm{~m} ; 8 \mathrm{f}$ \\
Hypertension & $6(54.5 \%)$ & $26(76.4 \%)$ & $13(86.6 \%)$ \\
Pulse pressure & $66 \pm 5$ & $68 \pm 5$ & $69 \pm 6$ \\
Hyperlipidemia & $7(63.6 \%)$ & $22(53.6 \%)$ & $9(60.0 \%)$ \\
Obesity & $2(18.1 \%)$ & $3(8.8 \%)$ & $2(13.3 \%)$ \\
Fam history CAD & $9(81.8 \%)$ & $3(8.8 \%)$ & $5(33.3 \%)$ \\
Active smoker & $/$ & $4(11.7 \%)$ & $4(3.7 \%)$
\end{tabular}

CAD - coronary artery disease

Table 3. Stepwise logistic regression analysis for predictors of myocardial ischemia

\begin{tabular}{|c|c|c|c|c|c|c|c|c|}
\hline & B & S.E. & Wald & df & Sign & OR & $95 \% \mathrm{Cl}$ & \\
\hline Male gender & 1.965 & 0.791 & 5.487 & 1 & 0.000 & 2.947 & 2.198 & 5.125 \\
\hline BPs & 1.042 & 0.633 & 6.285 & 1 & 0.000 & 3.126 & 1.934 & 3.857 \\
\hline LDL & 1.891 & 1.157 & 7.163 & 1 & 0.000 & 2.880 & 1.756 & 3.668 \\
\hline C-IMT & 1.945 & 1.264 & 6.261 & 1 & 0.000 & 3.811 & 2.251 & 5.963 \\
\hline
\end{tabular}

BPs — systolic blood pressure; LDL — low density holesterol; c-IMT — carotid intima media thickness 
Table 5. The association between the carotid artery ultrasound parameters and the severity of SPECT myocardial ischemia in the cohort study

$\begin{array}{lcccc}\text { Variables } & \begin{array}{c}\text { Normal MPS } \\ \text { result } \\ \mathrm{n}=42\end{array} & \begin{array}{c}\text { Mild } \\ \text { ischemia } \\ \mathrm{n}=6\end{array} & \begin{array}{c}\text { Moderate } \\ \text { ischemia }\end{array} & \begin{array}{c}\text { Severe } \\ \text { ischemia }\end{array} \\ & 0.71 \pm 0.20 & 0.81 \pm 0.1 & 0.90 \pm 0.1 & 1.00 \pm 0.2 \\ \text { C-IMT } & 11(21.4 \%) & 2 / 6 & 3 / 7 & 3 / 5\end{array}$

C-IMT — carotid artery intima media thickness

Our study showed the prevalence of moderate and severe ischemia increased from $4.5 \%$ in patients with normal c-IMT values to $25 \%$ in patients with increased mean c-IMT values (Figure 2).

\section{Parameters predictors of increased c-IMT and carotid plaques}

Multivariate analysis showed obesity, low HDL and diastolic blood pressure independent predictors for increased mean c-IMT (Table 6) and non-HDL, age and pulse pressure as independent predictors for presence of carotid plaques (Table 7).

\section{Discussion}

Silent myocardial ischemia - SMI is the most common manifestation of ischemic heart disease - IHD. $75 \%$ of ischemic episodes are asymptomatic and $48 \%$ of these patients have only one or even none of the traditional risk factors [1-3]. Diabetes, hypertension, previous myocardial infarction, surgical revascularization and advanced age are presumed risk factors for silent ischemia, even though many patients do not have any apparent risk factors and seem to be totally asymptomatic $[4,6]$. In clinical practice it is difficult to identify subjects with a high risk of SMI among an asymptomatic population. The rationale for identifying $\mathrm{SMI}$ is that this disorder is associated with an increase in coronary risk. All this data urge a demand for effective screening to reduce the risk of unexpected coronary events by sophisticating individual risk. The biggest scientific evidence for the prevalence of SMI comes from MPI studies, which report the SMI prevalence of $15-63 \%$ depending of the criteria used, study population and protocols $[4,6]$.

The ongoing debate on the prognostic relevance of SMI screening in the era of modern intensive medical therapy caused many controversies among consensus documents of the American Diabetes Association (ADA) and European Society of Cardiology (ESC) and European Association for the study of Diabetes (EASD) concerning the screening for $\mathrm{CAD}$ in asymptomatic diabetic population. CAD is the leading cause of mortality and morbidity in DMT2 patients [5]. The asymptomatic phase of CAD in DMT2 patients causes difficulty in CAD detection. There are several prospective studies that have shown the value of SPECT MPI in detection of myocardial ischemia, with the prevalence of moderate to severe grade ischemia in 6-8\% patients. Our study includes high number of patients with hypertension and hyperlipidemia and lower proportion of obese patients (11.6\%). Myocardial ischemia was found in 18 patients (30\%) in our study. These results correspond well with those reported in the literature [14-16]. Mild ischemia was found in 6 patients (10\%), moderate ischemia in 7 patients (11.6\%) and severe ischemia in 5 patients (8.3\%). Our multivariate regression analysis show independent predictors for SMI male gender, systolic blood pressure and LDL cholesterol. In DIAD study, independent predictive factor was diabetic neuropathy [10]. Zellweger et al., who analyzed 3664 asymptomatic patients without known CAD history using SPECT MPI, observed that $7.5 \%$ ischemic myocardium was related to high risk factors [15]. Scholte et al., found independent predictors of ischemia smoking, diabetes duration and cholesterol/HDL ratio [16]. This discrepancy in SMI predictors might be caused by the other factors characteristic for the development of diabetic micro vascular and macro vascular complications, such as genetic and still understudied markers.

Ultrasonographic measurement of carotid IMT allows the noninvasive and early detection of atherosclerotic changes and is used as a noninvasive end point for assessing the progression and regression of atherosclerosis in clinical trials [18]. In patients with a history of cardiovascular event, IMT correlates linearly with overall and cardiac mortality [20].

Table 6. Stepwise logistic regression analysis for predictors of increased c-IMT

\begin{tabular}{|c|c|c|c|c|c|c|c|c|}
\hline & B & S.E. & Wald & df & Sign & OR & $95 \% \mathrm{Cl}$ & \\
\hline Obesity & 1.835 & 0.251 & 5.260 & 1 & 0.000 & 2.931 & 1.688 & 5.127 \\
\hline Low HDL & 1.122 & 0.423 & 7.479 & 1 & 0.000 & 2.486 & 1.734 & 3.566 \\
\hline $\mathrm{BPd}$ & 1.343 & 1.587 & 7.325 & 1 & 0.002 & 3.127 & 1.946 & 4.198 \\
\hline
\end{tabular}

Abbreviations: BPd — diastolic blood pressure; $\mathrm{HDL}$ — high density lipoprotein

Table 7. Stepwise logistic regression analysis for predictors of carotid plaques

\begin{tabular}{|c|c|c|c|c|c|c|c|c|}
\hline & B & S.E. & Wald & df & Sign & OR & $95 \% \mathrm{Cl}$ & \\
\hline Non-HDL & 1.285 & 0.321 & 4.470 & 1 & 0.000 & 3.132 & 2.398 & 6.158 \\
\hline Age & 1.042 & 0.543 & 6.599 & 1 & 0.000 & 2.786 & 1.721 & 3.567 \\
\hline PP & 1.567 & 1.792 & 7.323 & 1 & 0.000 & 2.937 & 1.954 & 3.268 \\
\hline
\end{tabular}

PP — pulse pressure; HDL — high density lipoprotein 
The presence of carotid artery plaques correlates with the presence and extent of significant CAD, older age and the presence of diabetes and left ventricular hypertrophy [21]. According to Boucher et al., IMT values $<0.55 \mathrm{~mm}$ are associated with a negative predictive value of $80 \%$ for abnormal SPECT results; in nonsmokers the value is $>90 \%$ [22]. In our study, the increased c-IMT was a strong predictor of the extent of myocardial ischemia. Normal C-IMT values were associated with a low risk of presence of abnormal scintigraphic result. Only three asymptomatic diabetic patients with normal CIMT values had severely abnormal perfusion (5\%). However, it must be underlined that our study group had mean 2 risk factors and may represent a more high-risk population than the general asymptomatic DMT2 patients. Our multivariable analysis shows independent predictors for the increased mean c-IMT obesity, obesity, low HDL cholesterol and diastolic blood pressure. Independent predictors for carotid plaques presence were age, non- HDL cholesterol and pulse pressure (PP). Previous studies reported PP as a predictor of acute coronary events and as an independent risk factor for stroke [21, 22]. Mean C-IMT value increased with the ischemia severity on MPI imaging. Our study confirms that the increased C-IMT is linked to an increased likelihood of CAD presence in asymptomatic DMT2 patients.

The Atherosclerosis Risk in Communities (ARIC) study reported statistically significant associations of change in CIMT with baseline diabetes, current smoking, HDL cholesterol, pulse pressure, white blood cell count and fibrinogen [32]. Data from the Rotterdam study indicated that moderate in severe progression of C-IMT was related to age, body mass index, male sex, current smoking, systolic blood pressure and the presence of hypertension [33].

Our study emphasizes the clinical value of SPECT myocardial imaging for detection of SMI in selected diabetes type 2 patients with at least two risk factors and increased c-IMT, which might influence further patient management.

\section{Study limitations}

The small sample we evaluated could represent a limitation of our study. However, our results could help to improve our understanding of the relationship between risk factors, carotid artery atherosclerosis and coronary function in asymptomatic DMT2 patients screened for myocardial ischemia.

\section{Conclusions}

Our study showed significant relation between c-IMT, risk factors and the presence of myocardial ischemia in asymptomatic patients with DMT2 detected by SPECT myocardial perfusion imaging. The high prevalence of SMI in high risk patients underlines the importance for CAD screening in highly selected DMT2 individuals, both for improving the quality of life and long term survival. Multivariate analysis showed male gender, systolic blood pressure, LDL cholesterol and increased mean c-IMT independent predictors for presence of myocardial ischemia.

\section{Disclosure}

No conflicts of interest declared.

\section{References}

1. Janand-Delenne B, Savin B, Habib G, et al. Silent myocardial ischemia in patients with diabetes: who to screen. Diabetes Care. 1999; 22(9): 1396-1400, doi: 10.2337/diacare.22.9.1396, indexed in Pubmed: 10480499.

2. Shah PK. Screening asymptomatic subjects for subclinical atherosclerosis: can we, does it matter, and should we? J. Am. Coll. Cardiol. 2010; 56(2): 98-105, doi: 10.1016/j.jacc.2009.09.081, indexed in Pubmed: 20620724.

3. Juutilainen A, Lehto S, Rönnemaa T, et al. Type 2 diabetes as a "coronary heart disease equivalent": an 18-year prospective population-based study in Finnish subjects. Diabetes Care. 2005; 28(12): 2901-2907, doi: 10.2337/diacare.28.12.2901, indexed in Pubmed: 16306552.

4. De Lorenzo A, Lima RSL, Siqueira-Filho AG, et al. Prevalence and prognostic value of perfusion defects detected by stress technetium-99m sestamibi myocardial perfusion single-photon emission computed tomography in asymptomatic patients with diabetes mellitus and no known coronary artery disease. Am J Cardiol. 2002; 90(8): 827-832, doi: 10.1016/s00029149(02)02702-9, indexed in Pubmed: 12372568.

5. Greenland P, Alpert JS, Beller GA, et al. American College of Cardiology Foundation, American Heart Association. 2010 ACCF/AHA guideline for assessment of cardiovascular risk in asymptomatic adults: a report of the American College of Cardiology Foundation/American Heart Association Task Force on Practice Guidelines. J Am Coll Cardiol. 2010; 56(25): e50-103, doi: 10.1016/j.jacc.2010.09.001, indexed in Pubmed: 21144964.

6. Mannami T, Konishi M, Baba S, et al. Prevalence of asymptomatic carotid atherosclerotic lesions detected by high-resolution ultrasonography and its relation to cardiovascular risk factors in the general population of a Japanese city: the Suita study. Stroke. 1997; 28(3): 518-525, doi: 10.1161/01. str.28.3.518, indexed in Pubmed: 9056605.

7. Hodis HN, Mack WJ, LaBree L, et al. The role of carotid arterial intima-media thickness in predicting clinical coronary events. Ann Intern Med. 1998; 128(4): 262-269, doi: 10.7326/0003-4819-128-4-199802150-00002, indexed in Pubmed: 9471928.

8. Simons P, Algra A, Bots ML, et al. Common Carotid Intima-Media Thickness and Arterial Stiffness : Indicators of Cardiovascular Risk in High-Risk Patie ntsThe SMART Study (Second Manifestations of ARTerial disease). Circulation. 1999; 100(9): 951-957, doi: 10.1161/01.cir.100.9.951.

9. Hachamovitch R, Berman DS, Kiat H, et al. Exercise myocardial perfusion SPECT in patients without known coronary artery disease: incremental prognostic value and use in risk stratification. Circulation. 1996; 93(5): 905-914, doi: 10.1161/01.cir.93.5.905, indexed in Pubmed: 8598081.

10. Wackers FJ, Young LH, Inzucchi SE, et al. Detection of Ischemia in Asymptomatic Diabetics Investigators. Detection of silent myocardial ischemia in asymptomatic diabetic subjects: the DIAD study. Diabetes Care. 2004; 27(8): 1954-1961, doi: 10.2337/diacare.27.8.1954, indexed in Pubmed: 15277423.

11. Faglia E, Favales F, Calia P, et al. Milan Study on Atherosclerosis and Diabetes (Mi SAD). Cardiac events in 735 type 2 diabetic patients who underwent screening for unknown asymptomatic coronary heart disease: 5-year follow-up report from the Milan Study on Atherosclerosis and Diabetes (MiSAD). Diabetes Care. 2002; 25(11): 2032-2036, doi: 10.2337/diacare.25.11.2032, indexed in Pubmed: 12401752.

12. Wang TJ, Nam BH, Wilson PWF, et al. Association of C-reactive protein with carotid atherosclerosis in men and women: the Framingham Heart Study. Arterioscler Thromb Vasc Biol. 2002; 22(10): 1662-1667, doi: 10.1161/01. atv.0000034543.78801.69, indexed in Pubmed: 12377746.

13. Acampa W, Petretta M, Evangelista L, et al. Myocardial perfusion imaging and risk classification for coronary heart disease in diabetic patients. The IDIS study: a prospective, multicentre trial. Eur J Nucl Med Mol Imaging. 2012; 39(3): 387-395, doi: 10.1007/s00259-011-1983-x, indexed in Pubmed: 22109666

14. Rajagopalan N, Miller TD, Hodge DO, et al. Identifying high-risk asymptomatic diabetic patients who are candidates for screening stress single-photon 
emission computed tomography imaging. J Am Coll Cardiol. 2005; 45(1): 43-49, doi: 10.1016/j.jacc.2004.06.078, indexed in Pubmed: 15629371.

15. Zellweger MJ, Maraun M, Osterhues $\mathrm{HH}$, et al. Progression to overt or silent CAD in asymptomatic patients with diabetes mellitus at high coronary risk: main findings of the prospective multicenter BARDOT trial with a pilot randomized treatment substudy. JACC Cardiovasc Imaging. 2014; 7(10): 1001-1010, doi: 10.1016/j.jcmg.2014.07.010, indexed in Pubmed: 25240454.

16. Scholte AJ, Schuijf JD, Kharagjitsingh AV, et al. Prevalence and predictors of an abnormal stress myocardial perfusion study in asymptomatic patients with type 2 diabetes mellitus. Eur J Nucl Med Mol Imaging. 2009; 36(4): 567-575, doi: 10.1007/s00259-008-0967-y, indexed in Pubmed: 18985347.

17. Alonso N, Traveset A, Rubinat E, et al. Type 2 diabetes-associated carotid plaque burden is increased in patients with retinopathy compared to those without retinopathy. Cardiovasc Diabetol. 2015; 14: 33, doi: 10.1186/s12933015-0196-1, indexed in Pubmed: 25856787.

18. Hodis HN, Mack WJ, LaBree L, et al. The role of carotid arterial intima-media thickness in predicting clinical coronary events. Ann Intern Med. 1998; 128(4): 262-269, doi: 10.7326/0003-4819-128-4-199802150-00002, indexed in Pubmed: 9471928

19. Mancini GB, Dahlöf B, Díez J. Surrogate markers for cardiovascular disease: structural markers. Circulation. 2004; 109(25 Suppl 1): IV22-IV30, doi: 10.1161/01.CIR.0000133443.77237.2f, indexed in Pubmed: 15226248.

20. Bonithon-Kopp C, Scarabin PY, Taquet A, et al. Risk factors for early carotid atherosclerosis in middle-aged French women. Arterioscler Thromb. 1991; 11(4): 966-972, doi: 10.1161/01.atv.11.4.966, indexed in Pubmed: 2065047.

21. Franklin SS, Khan SA, Wong ND, et al. Is pulse pressure useful in predicting risk for coronary heart Disease? The Framingham heart study. Circulation. 1999; 100(4): 354-360, doi: 10.1161/01.cir.100.4.354, indexed in Pubmed: 10421594

22. Benetos A, Rudnichi A, Safar M, et al. Pulse pressure and cardiovascular mortality in normotensive and hypertensive subjects. Hypertension. 1998; 32(3): 560-564, doi: 10.1161/01.hyp.32.3.560, indexed in Pubmed: 9740626.

23. Nossen JM, Vierzigmann T, Lang E. Calcified plaques of extracranial carotid arteries and left ventricular geometry as predictors of coronary artery disease. Med Klin (Munich). 2003; 98(2): 72-78, doi: 10.1007/s00063-0031229-1, indexed in Pubmed: 12601531.
24. Boucher B, Cerisier A, Bouchou K, et al. [Silent myocardial ischemia in type 2 diabetes. Predictive value of intima-media thickness]. Presse Med. 2002; 31(5): 218-222, doi: 10.1016/s1544-8800(07)70392-5, indexed in Pubmed: 11878140

25. Djaberi R, Schuijf JD, Jukema JW, et al. Increased carotid intima-media thickness as a predictor of the presence and extent of abnormal myocardial perfusion in type 2 diabetes. Diabetes Care. 2010; 33(2): 372-374, doi: 10.2337/dc09-1301, indexed in Pubmed: 19918012.

26. Chambless LE, Folsom AR, Davis $\mathrm{V}$, et al. Risk factors for progression of common carotid atherosclerosis: the Atherosclerosis Risk in Communities Study, 1987-1998. Am J Epidemiol. 2002; 155(1): 38-47, doi: 10.1093/aje/155.1.38, indexed in Pubmed: 11772783.

27. van der Meer IM, Iglesias del Sol A, Hak AE, et al. Risk factors for progression of atherosclerosis measured at multiple sites in the arterial tree: the Rotterdam Study. Stroke. 2003; 34(10): 2374-2379, doi: 10.1161/01. STR.0000088643.07108.19, indexed in Pubmed: 12947155.

28. Kuller L, Borhani N, Furberg C, et al. Prevalence of subclinical atherosclerosis and cardiovascular disease and association with risk factors in the Cardiovascular Health Study. Am J Epidemiol. 1994; 139(12): 1164-1179, doi: 10.1016/j.atherosclerosis.2005.09.015, indexed in Pubmed: 8209875.

29. Bosevski M. Carotid Artery Disease in Diabetic Patients. PRILOZI. 2015; 35(3), doi: 10.1515/prilozi-2015-0019.

30. Bosevski M, Peovska I. Non-invasive imaging of diabetic vascular disease. Nuc Med Rev Cent East Eur. 2010; 13(1): 39-47, indexed in Pubmed: 21154315.

31. Sibal L, Agarwal SC, Home PD. Carotid intima-media thickness as a surrogate marker of cardiovascular disease in diabetes. Diabetes Metab Syndr Obes. 2011; 4: 23-34, doi: 10.2147/DMSO.S8540, indexed in Pubmed: 21448319.

32. Folsom AR, Eckfeldt JH, Weitzman S, et al. Relation of carotid artery wall thickness to diabetes mellitus, fasting glucose and insulin, body size, and physical activity. Atherosclerosis Risk in Communities (ARIC) Study Investigators. Stroke. 1994; 25(1): 66-73, doi: 10.1161/01.str.25.1.66, indexed in Pubmed: 8266385

33. Bots ML, Hoes AW, Koudstaal PJ, et al. Common carotid intima-media thickness and risk of stroke and myocardial infarction: the Rotterdam Study. Circulation. 1997; 96(5): 1432-1437, doi: 10.1161/01.cir.96.5.1432, indexed in Pubmed: 9315528 